\title{
Breast ductal carcinoma in situ carry mutational driver events representative of invasive breast cancer
}

Jia-Min B Pang ${ }^{1,2}$, Peter Savas ${ }^{3}$, Andrew P Fellowes ${ }^{1}$, Gisela Mir Arnau ${ }^{4}$, Tanjina Kader ${ }^{5,6}$, Ravikiran Vedururu ${ }^{1}$, Chelsee Hewitt ${ }^{1}$, Elena A Takano ${ }^{1}$, David J Byrne ${ }^{1}$, David YH Choong ${ }^{1}$, Ewan KA Millar ${ }^{7,8,9,10}$, C Soon Lee ${ }^{10,11,12}$, Sandra A O'Toole ${ }^{7,11,13}$, Sunil R Lakhani ${ }^{14,15}$, Margaret C Cummings ${ }^{14,15}$, G Bruce Mann ${ }^{16,17}$, Ian G Campbell ${ }^{5,6}$, Alexander Dobrovic ${ }^{18}$, Sherene Loi ${ }^{3,5}$, Kylie L Gorringe ${ }^{2,5,6,19}$ and Stephen B Fox ${ }^{1,2,5,19}$

${ }^{1}$ Department of Pathology, Peter MacCallum Cancer Centre, Grattan Street, Melbourne, VIC, Australia; ${ }^{2}$ Department of Pathology, University of Melbourne, Grattan Street, Parkville, Melbourne, VIC, Australia; ${ }^{3}$ Division of Research and Cancer Medicine, Peter MacCallum Cancer Centre, Grattan Street, Melbourne, VIC, Australia; ${ }^{4}$ Molecular Genomics Core, Peter MacCallum Cancer Centre, Grattan Street, Melbourne, VIC, Australia; ${ }^{5}$ Sir Peter MacCallum Department of Oncology, University of Melbourne, Grattan Street, Parkville, Melbourne, VIC, Australia; ${ }^{6}$ Cancer Genomics Program, Peter MacCallum Cancer Centre, Grattan Street, Melbourne, VIC, Australia; ${ }^{7}$ Translational Breast Cancer Research, The Kinghorn Cancer Centre and Garvan Institute of Medical Research, Darlinghurst, NSW, Australia; ${ }^{8}$ Department of Anatomical Pathology, South Eastern Area Pathology Service, St George Hospital, Kogarah, NSW, Australia; ${ }^{9}$ School of Medical Sciences, University of New South Wales, Kensington, NSW, Australia; ${ }^{10}$ Discipline of Pathology, School of Medicine, University of Western Sydney, Campbelltown, NSW, Australia; ${ }^{11}$ Department of Tissue Pathology and Diagnostic Oncology, Royal Prince Alfred Hospital, Camperdown, NSW, Australia; ${ }^{12}$ Cancer Pathology, Bosch Institute, University of Sydney, Camperdown, NSW, Australia; ${ }^{13}$ Sydney Medical School, University of Sydney, Camperdown, NSW, Australia; ${ }^{14}$ School of Medicine and UQ Centre for Clinical Research, The University of Queensland, Brisbane, Australia; ${ }^{15}$ Pathology Queensland, The Royal Brisbane and Women's Hospital, Brisbane, Australia; ${ }^{16}$ Breast Service, The Royal Melbourne and Royal Women's Hospitals, Parkville, VIC, Australia; ${ }^{17}$ Department of Surgery, University of Melbourne, Grattan Street, Parkville, Melbourne, VIC, Australia and ${ }^{18}$ Translational Genomics \& Epigenomics Laboratory, Olivia Newton-John Cancer Research Institute, Heidelberg, VIC, Australia

The spectrum of genomic alterations in ductal carcinoma in situ (DCIS) is relatively unexplored, but is likely to provide useful insights into its biology, its progression to invasive carcinoma and the risk of recurrence. DCIS $(n=20)$ with a range of phenotypes was assessed by massively parallel sequencing for mutations and copy number alterations and variants validated by Sanger sequencing. PIK3CA mutations were identified in 11/20 (55\%), TP53 mutations in 6/20 (30\%), and GATA3 mutations in 9/20 (45\%). Screening an additional 91 cases for GATA3 mutations identified a final frequency of $27 \%(30 / 111)$, with a high proportion of missense variants $(8 / 30)$. TP53 mutations were exclusive to high grade DCIS and more frequent in PR-negative tumors compared with PR-positive tumors $(P=0.037)$. TP53 mutant tumors also had a significantly higher fraction of the genome altered by copy number than wild-type tumors $(P=0.005)$, including a significant positive association with amplification or gain of ERBB2 $(P<0.05)$. The association between TP53 mutation and ERBB2 amplification was confirmed in a wider DCIS cohort using p53 immunohistochemistry as a surrogate marker for TP53 mutations $(P=0.03)$. RUNX1 mutations and MAP2K4 copy number loss were novel findings in DCIS. Frequent copy number alterations included gains on 1q, 8q, 17q, and 20q and losses on 8p, 11q, 16q, and 17p. Patterns of genomic alterations observed in DCIS were similar to those previously reported for invasive breast cancers, with all DCIS having at least one bona fide breast cancer driver event. However, an increase in GATA3 mutations and fewer copy number changes were noted in DCIS compared with invasive carcinomas. The role of such alterations as prognostic and predictive biomarkers in DCIS is an avenue for further investigation. Modern Pathology (2017) 30, 952-963; doi:10.1038/modpathol.2017.21; published online 24 March 2017

Correspondence: Dr KL Gorringe, PhD, Cancer Genomics Program, Peter MacCallum Cancer Centre, Grattan Street, Melbourne, VIC 3000, Australia. E-mail: kylie.gorringe@petermac.org

${ }^{19}$ These authors contributed equally to this work.

Received 5 January 2017; revised 11 February 2017; accepted 19 February 2017; published online 24 March 2017 
The mutational landscape of invasive breast carcinoma has been extensively documented in recent landmark studies. ${ }^{1-5}$ However, little is known about the mutational profile of ductal carcinoma in situ (DCIS). Studies have addressed mutations of individual genes ${ }^{6-13}$ but until very recently, assessment of a broad panel of genes in DCIS has not been performed, largely due the challenges in obtaining DNA from DCIS cases compatible with highly multiplexed methodologies. To date, whole genome or whole exome sequencing has been performed in only 45 published DCIS cases in total, all using DNA from fresh frozen tissue. ${ }^{4,14,15}$ Therefore, the cases used to date have been highly selected, derived from large mass forming tumors with adequate sufficient tissue to spare after diagnostic samples were taken.

To document the mutational profile of DCIS we performed massive parallel sequencing of a comprehensive panel of cancer-related genes on formalinfixed, paraffin-embedded derived DNA from 20 DCIS tumors representing a full range of phenotypes. Our aims were to (1) document the mutational landscape of DCIS of different grades, hormone receptor and ERBB2 status, (2) to identify differences between invasive and pre-invasive disease, (3) assess whether particular genomic alterations correlate with clinicopathological parameters, and (4) determine whether genomic profiling of DCIS can be implemented in the routine diagnostic setting.

\section{Materials and methods}

\section{Clinicopathological Parameters of Patients and Tumors}

Twenty DCIS cases were selected to represent a range of tumor characteristics (Table 1) and to have sufficient DNA quantity and quality to ensure successful sequencing. The median age of patients at surgery was 56.5 years (range 29-90 years, mean 56.5 years). Ten cases were of high nuclear grade, seven of intermediate nuclear grade, and three of low nuclear grade. Sixteen cases were estrogen receptor (ER)-positive, 14 progesterone receptor (PR)-positive, and five cases were HER2 amplified. No long term clinical outcome data was available for these cases. Five cases had matched normal DNA available derived from adjacent breast tissue. Additional cases for GATA3 sequencing were obtained from Royal Melbourne Hospital as previously described. ${ }^{16}$ Approval for the study was obtained from the ethics committee of Peter MacCallum Cancer Centre (project numbers 02/26, 10/16, and 00/81).

\section{Sample Processing and Sequencing}

Areas of DCIS were microdissected from formalin-fixed paraffin embedded sections and DNA extracted using the QIAamp DNA Blood Mini Kit (Qiagen, Hilden, Germany) according to the manufacturer's instructions.
A total of $500 \mathrm{ng}$ per sample was used for sequencing. The KAPA Hyper Prep Kit (Kapa Biosystems, Wilmington, MA, USA) was used for library preparation with Agencourt AMPure XP beads (Beckman Coulter, Brea, CA, USA) for library clean-up. Target capture was performed using the SureSelectXT Target Enrichment System (Agilent Technologies, Santa Clara, CA, USA), targeting the exons of 107 cancer-related genes (Supplementary Methods), including 61 genes of specific relevance to breast cancer, covering a total of $360 \mathrm{~kb}$. Captured libraries were sequenced using a NextSeq500 sequencer (Illumina, San Diego, CA, USA).

\section{Identification and Filtering of Variants}

Sequence alignment and variant calling was performed by aligning the sequencer output to the reference genome using Burrows-Wheeler Aligner software $^{17}$ and variants were called using the SNPPET algorithm provided in the Agilent SureCall software package (Agilent Technologies). The variants identified by the Agilent SureCall algorithm were filtered for non-synonymous variants in exonic or essential splice site locations with a variant allele frequency of at least $10 \%$. The allele frequency cut-off of $10 \%$ was chosen to minimize inclusion of sequencing artefacts related to formalin fixation. ${ }^{18}$ As no matched normal DNA was submitted for sequencing, the 1000 Genomes database was used to exclude potential germline variants, which may represent non-pathogenic genetic variation in the population. ${ }^{19}$ The sequencing reads in regions containing variants were then visually inspected using Integrative Genomics Viewer to exclude potentially artefactual variants, such as those occurring in variant-rich regions or variants identified exclusively at read ends.

\section{Validation of Variants by Sanger Sequencing}

The variants identified by massively parallel sequencing were validated by Sanger sequencing. Normal DNA, where available $(n=5)$, was also subjected to Sanger sequencing alongside matching DCIS samples. Additional samples were sequenced for GATA3 exons 5 and 6 using different primers with greater exon coverage. Sanger sequencing primers were designed using the Primer 3 tool. ${ }^{20,21}$ Target sequences were amplified using primers and conditions listed in Supplementary Methods. The BigDye Terminator system (Applied Biosystems) was used for sequencing on a 3730 DNA Analyzer (Applied Biosystems). The sequencer output was viewed using Sequencer 4.8 software (Gene Codes Corporation, Ann Arbor, MI, USA) or Geneious (Biomatters, Auckland, New Zealand).

\section{p53 Immunohistochemistry}

Immunohistochemistry for p53 was performed using a Ventana BenchMark Ultra (Roche Diagnostics, 
USA) on $3 \mu \mathrm{m}$ formalin-fixed paraffin embedded tissue sections of tissue microarrays containing $0.5-2$ mm cores of DCIS with up to eight-fold redundancy as described previously. ${ }^{22,23}$ Antigen retrieval was performed in a high $\mathrm{pH}$ Ultra cell conditioning solution (CC1, Roche Diagnostics) for $32 \mathrm{~min}$ at $100^{\circ}$ C. Sections were incubated with the p53 antibody (Novocastra Liquid Mouse Monoclonal DO-7, Leica Biosystems) at $1 / 100$ for $24 \mathrm{~min}$ at $36^{\circ} \mathrm{C}$. The Onboard detection system, OptiView Universal DAB Detection Kit (Roche Diagnostics) was used in accordance with the manufacturer's instructions.

p53 immunohistochemical nuclear reactivity was scored for intensity $(0=$ no reactivity, $1=$ weak, $2=$ moderate, $3=$ strong reactivity) and percentage of tumor cells positive to the closest $5 \%$. Absence of p53 nuclear reactivity or nuclear reactivity in $\geq 60 \%$ of tumor cells was considered to be an abnormal pattern and suggestive of TP53 mutation. ${ }^{24}$ For cases represented by more than one tumor core, absence of p53 nuclear reactivity in all cores or $\geq 60 \%$ nuclear reactivity in any core was considered an abnormal pattern.

\section{Copy Number Analysis}

Copy number was generated using the CopywriteR tool in $\mathrm{R}^{25}$ using a normal DNA sample that was included in the same sequencing batch as the baseline control. Copy number data from invasive breast carcinomas was downloaded as Level 3 data from the TCGA Data portal. Data were imported to Nexus (BioDiscovery) with thresholds of gain and loss of $\pm 0.2 \log _{2}$ ratio. Fraction of the genome altered was calculated as the percentage of the genome in base pairs affected by gain or loss. Mutation data from TCGA was obtained from the cBio Portal. ${ }^{26}$ The results published here are, in part, based upon data generated by TCGA project established by the NCI and NHGRI. Information about TCGA and the investigators and institutions who constitute the TCGA research network can be found at http://cancergenome.nih.gov.

\section{Results}

\section{Sequence Variants Detected}

The median number of reads per sample was 20,054,319 (range $17504368-24942$ 911; mean 20300466 ), with a median $16.0 \%$ being duplicate reads (range 10.2-27.7\%; mean 16.6\%). Total percentage mapped reads on average was $99.7 \%$ (median 99.7\%; range 99.6-99.8\%; mean 99.7\%), and $60.1 \%$ were on-target reads (median $60.1 \%$; range $51.8-66.1 \%$; mean $60.1 \%$ ). The median readdepth over the target regions was 1692 reads (range 1325-2031; mean 1651) and the average percentage of target bases with over 1000 reads was $99.1 \%$. These parameters indicate very good technical 
performance of the assay, which is expected to yield good quality and reliable data.

There were a total of 11145 variants in 103 genes identified by the Agilent SureCall algorithm. The median number of variants per sample was 600.5 (range 150-652; mean 557.3 variants per sample). Filtering based on inspection of the sequencing reads and the 1000 Genomes data set resulted in a reduction in the number of variants $(81.9 \%$ and $73.8 \%$ of variants, respectively), suggesting that most of the variants in the target regions were likely sequencing artefacts or single nucleotide polymorphisms. After filters were applied, 52 candidate somatic variants in 25 genes remained and were validated by Sanger sequencing (Table 2).

The majority of variants identified were validated by Sanger sequencing $(48 / 50,96 \%)$, apart from those occurring in FGFR1 and PALB2 (one variant each). It is unclear why these failed to validate, although with allele frequencies of 0.21 and 0.12 , respectively, it is possible these were below the sensitivity of Sanger sequencing. The GATA3 variants of two samples (chr10:8111479insC, allele frequency 0.26 and chr10:8115709_8115711delGAC, allele frequency 0.35) were not validated due to insufficient DNA. However, variants occurring in the same regions as these two GATA3 variants were validated in other samples, giving confidence to the sequencing results (Table 2). Four variants in two patients were present in both the DCIS and matching normal DNA samples (Table 2). It is possible that other very rare germline variants remain in the validated variants, as some samples did not have matching normal DNA for comparison.

\section{Mutated Genes in DCIS}

Following Sanger validation there were a total of 46 variants in 19 genes over 20 samples. The cohort had a median of two variants per sample (range 1-4 variants per sample; mean 2.3 variants per sample). Forty variants $(87 \%)$ were located in exons and the remaining six $(13 \%)$ at essential splice sites. Similar to previous studies of DCIS, ${ }^{14,15}$ single-nucleotide substitutions were the most frequent mutation type detected $(33 / 46,71.7 \%)$, with the most frequent substitution being $\mathrm{C}>\mathrm{T}: \mathrm{G}>\mathrm{A} \quad(14 / 33,42.4 \%)$ (Figure 1).

PIK3CA was the most frequently mutated gene, harboring 12 mutations in 11 cases (11/20, 55\%). All the PIK3CA mutations were missense mutations. There were ten known activating PIK3CA mutations, five in the helical domain (one E542K, one E545K, and three E545A mutations) and five located in the kinase domain (one H1047Y and four H1047R mutations). In addition there was a E542V mutation (helical domain), predicted to be damaging and deleterious by the SIFT ${ }^{27}$ and PROVEAN ${ }^{28}$ algorithms, respectively. One mutation (E726K) was predicted to be non-deleterious. ${ }^{27,28}$ One case had co-existing E542K and E545A mutations, which were mutually exclusive on the sequencing reads with differing allele frequencies $(0.23$ and 0.10 , respectively), suggesting that these mutations were present in separate clones rather than being mutations on separate alleles of the same clone.

The next most commonly mutated genes were GATA3 (9/20, 45\%) and TP53 (6/20, 30\%). In contrast to PIK3CA, only one of the nine GATA3 mutations was a missense mutation, the remainder being four splice site mutations, three frameshift insertions, and one in-frame deletion. Both the missense mutation and in-frame deletion were predicted to be damaging and deleterious by the SIFT $^{27}$ and PROVEAN ${ }^{28}$ algorithms, respectively. All but one of the mutations affected exons 5 and 6 , consistent with the mutation types previously reported for this gene. ${ }^{5}$ Of the TP53 mutations, three were missense mutations, two splice site mutations and one was a frameshift deletion. The missense and splice site mutations are known deleterious mutations recorded in the IARC database. ${ }^{29}$ Co-existing mutations of GATA3 and TP53 were present in one case, of PIK3CA and TP53 in three cases, and of PIK3CA and GATA3 in four cases (Figure 2). RUNXI (Figure 3) and TSC2 were mutated in two cases each $(2 / 20,10 \%)$. The remaining genes were mutated in one case each $(1 / 20,5 \%$, Table 2 and Figure 2$)$. Of note, one case (P124) had two truncating mutations in PIK3R1 suggestive of bi-allelic inactivation.

The proportion of GATA3 mutated cases observed $(45 \%)$ was considerably higher than that observed in studies of invasive breast cancer (4-22\%). $4,5,30,31$ We therefore evaluated GATA3 mutation status in a further 91 pure DCIS cases using Sanger sequencing of exons 5 and 6 (as DNA was limited). Nonsynonymous or splice region mutations were identified in $21 / 91$ cases (23\%, Supplementary Table 1), for a final frequency of $30 / 111$ cases $(27 \%)$. This frequency is significantly higher than observed in invasive breast cancer (TCGA 54/507, $P<0.0001$, Fisher's exact test), and remains significantly more when only those with known ER positive status are considered (25/70 (36\%) compared with 54/390 $(14 \%)$ in TCGA, $P<0.0001)$. We observed a higher proportion of missense mutations in our cohort than expected compared with invasive cohorts $(8 / 30)$, nevertheless it is possible these are rare polymorphisms not represented in any existing databases, as no matching normal DNA was available to remove germline variants. However, even considering only overtly deleterious mutations (splice site and truncating), we still observed a significantly higher frequency than expected from TCGA data (19\% all, $P=0.02,27 \%$ ER-positive only, $P=0.007$ ).

We hypothesized that GATA3 mutations might predict a better outcome, however, within ER positive cases treated by wide local excision, there was no association with recurrence $(P=0.20$, Cox log-rank test), although the power in this analysis 
Table 2 Variants (filtered) detected by sequencing panel

\begin{tabular}{|c|c|c|c|c|c|c|c|}
\hline Gene & Genomic alteration (GRCg37/hg19) & Transcript & Transcript alteration & Protein alteration & Case & Validation & Germline result \\
\hline ARID1A & $\operatorname{chr} 1: 27100375 \mathrm{C}>\mathrm{T}$ & NM_139135 & c. $4087 \mathrm{C}>\mathrm{T}$ & p.Q1363X & P53 & Validated & No matched normal \\
\hline ATM & chr11:108196837G $>A$ & NM_000051 & c. $6860 \mathrm{G}>\mathrm{A}$ & p.G2287E & P122 & Validated & No matched normal \\
\hline BRCA2 & $\operatorname{chr} 13: 32899219 A>G$ & NM_000059 & c. $323 \mathrm{~A}>\mathrm{G}$ & p.N108S & $\mathrm{P} 43$ & Validated & Present in normal \\
\hline$C B F B$ & chr16:67100619T $>$ G & NM_001755 & c. $317 \mathrm{~T}>\mathrm{G}$ & p.V106G & P121 & Validated & No matched normal \\
\hline CDH1 & chr16:68844136G $>A$ & NM_004360 & c. $724 \mathrm{G}>\mathrm{A}$ & p.V242I & $\mathrm{P} 43$ & Validated & Present in normal \\
\hline CDKN2A & chr9:21994285C $>$ A & NM_058195 & c.G46T & p.G16C & P122 & Validated & No matched normal \\
\hline ERBB2 & chr17:37882896G $>A$ & NM_004448 & c. $2954 \mathrm{G}>\mathrm{A}$ & p.R985H & $\mathrm{P} 66$ & Validated & No matched normal \\
\hline ERCC2 & chr19:45872243G $>A$ & NM_000400 & c. $191 \mathrm{C}>\mathrm{T}$ & p.P64L & P44 & Validated & Present in normal \\
\hline FGFR1 & chr8:38287292T $>$ C & NM_015850 & с. $266 \mathrm{~A}>\mathrm{G}$ & p.Q89R & P66 & Not detected & No matched normal \\
\hline FGFR3 & chr4:1801503G > A & NM_000142 & c. $409 \mathrm{G}>\mathrm{A}$ & p.G137R & P43 & Validated & Present in normal \\
\hline FGFR4 & chr5:176517459C $>\mathrm{T}$ & XM_005265838 & c. $160 \mathrm{C}>\mathrm{T}$ & p.R54C & P122 & Validated & No matched normal \\
\hline GATA3 & chr10:8106075insC & NM_001002295 & c.898dupC & p.L301Pfs ${ }^{*} 3$ & P64 & Validated & No matched normal \\
\hline GATA3 & chr10:8111433-8111434delCA & NM_001002295 & c.925-3_925-2delCA & splice site & P121 & Validated & No matched normal \\
\hline GATA3 & chr10:8111433-8111434delCA & NM_001002295 & c.925-3_925-2delCA & splice site & P44 & Validated & Absent in normal \\
\hline GATA3 & chr10:8111433-8111434delCA & NM_001002295 & c.925-3_925-2delCA & splice site & P81 & Validated & No matched normal \\
\hline GATA3 & chr10:8111479insC & NM_001002295 & c.968dupC & p.T324Hfs*29 & P66 & Insufficient DNA & No matched normal \\
\hline GATA3 & chr10:8115700A >C & NM_001002295 & c. $1051-2 \mathrm{G}>\mathrm{A}$ & splice site & P89 & Validated & Absent in normal \\
\hline GATA3 & chr10:8115709-8115711delGAC & NM_001002295 & c.1058_1060delGAC & p.R353_P354delinsT & P92 & Insufficient DNA & No matched normal \\
\hline GATA3 & $\operatorname{chr} 10: 8115712 \mathrm{C}>\mathrm{A}$ & NM_001002295 & c. $1061 \mathrm{C}>\mathrm{A}$ & p.P354H & P45 & Validated & No matched normal \\
\hline GATA3 & chr10:8115950insACACCACCССТ & NM_001002295 & c.1299_1300insACACCACCCCT & p.H434Tfs*46 & P79 & Validated & No matched normal \\
\hline$N B N$ & chr8:90967716G $>$ A & NM_002485 & c. $1192 \mathrm{C}>\mathrm{T}$ & p.Q398X & P64 & Validated & No matched normal \\
\hline NF2 & $\operatorname{chr} 22: 30061039 \mathrm{C}>\mathrm{T}$ & NM_000268 & c. $871 \mathrm{C}>\mathrm{T}$ & p.R291C & P54 & Validated & No matched normal \\
\hline$P A L B 2$ & chr16:23646349T $>$ A & NM_024675 & c. $1518 \mathrm{~A}>\mathrm{T}$ & p.Q506H & P56 & Not detected & No matched normal \\
\hline РIКЗСА & chr3:178936082G > A & NM_006218 & c. $1624 \mathrm{G}>\mathrm{A}$ & p.E542K & P125 & Validated & No matched normal \\
\hline РІКЗСА & $\operatorname{chr} 3: 178936083 \mathrm{~A}>\mathrm{T}$ & NM_006218 & c. $1625 \mathrm{~A}>\mathrm{T}$ & p.E542V & P53 & Validated & No matched normal \\
\hline РІКЗСА & chr3:178936091G $>A$ & NM_006218 & c. $1633 \mathrm{G}>\mathrm{A}$ & p.E545K & P13 & Validated & Absent in normal \\
\hline РIK3CA & $\operatorname{chr} 3: 178936092 \mathrm{~A}>\mathrm{C}$ & NM_006218 & c. $1634 \mathrm{~A}>\mathrm{C}$ & p.E545A & P125 & Validated & No matched normal \\
\hline РІКЗСА & $\operatorname{chr} 3: 178936092 \mathrm{~A}>\mathrm{C}$ & NM_006218 & c. $1634 \mathrm{~A}>\mathrm{C}$ & p.E545A & P89 & Validated & Absent in normal \\
\hline РIК $3 C A$ & $\operatorname{chr} 3: 178936092 \mathrm{~A}>\mathrm{C}$ & NM_006218 & c. $1634 \mathrm{~A}>\mathrm{C}$ & p.E545A & P92 & Validated & No matched normal \\
\hline РIK3CA & chr3:178938934G $>A$ & NM_006218 & c. $2176 \mathrm{G}>\mathrm{A}$ & p.E726K & P54 & Validated & No matched normal \\
\hline РIKЗСA & chr3:178952084C $>\mathrm{T}$ & NM_006218 & c. $3139 \mathrm{C}>\mathrm{T}$ & p.H1047Y & P64 & Validated & No matched normal \\
\hline PIK $3 C A$ & chr3:178952085A $>G$ & NM_006218 & c. $3140 \mathrm{~A}>\mathrm{G}$ & p.H1047R & P121 & Validated & No matched normal \\
\hline PIKЗCA & $\operatorname{chr} 3: 178952085 \mathrm{~A}>\mathrm{G}$ & NM_006218 & c. $3140 \mathrm{~A}>\mathrm{G}$ & p.H1047R & P19 & Validated & No matched normal \\
\hline PIK $3 C A$ & chr3:178952085A $>$ G & NM_006218 & c. $3140 A>G$ & p.H1047R & P43 & Validated & Absent in normal \\
\hline PIK $3 C A$ & chr3:178952085A $>G$ & NM_006218 & c. $3140 \mathrm{~A}>\mathrm{G}$ & p.H1047R & P85 & Validated & No matched normal \\
\hline PIK3R1 & chr5:67576453-67576453delA & NM_181523 & c.732delA & p.K245Nfs*15 & P124 & Validated & No matched normal \\
\hline PIK3R1 & chr5:67591114-67591117delCCTT & NM_181504 & c.897_900del & p.D299Efs* 4 & P124 & Validated & No matched normal \\
\hline PTCH1 & chr9:98241349G > A & NM_000264 & c. $114 \overline{8} \mathrm{C}>\mathrm{T}$ & p.S383L & P27 & Validated & No matched normal \\
\hline RAD51D & chr17:33446607-33446608delCA & NM_002878 & c.25_26del & p.C9Pfs* 61 & P92 & Validated & No matched normal \\
\hline RUNX1 & chr21:36206724insG & NM_001001890 & c. $70 \overline{6}$ dupC & p.Q237Sfs*336 & P89 & Validated & Absent in normal \\
\hline RUNX1 & $\operatorname{chr} 21: 36252866 \mathrm{G}>\mathrm{C}$ & NM_001001890 & c. $415 \mathrm{C}>\mathrm{G}$ & p.R139G & P44 & Validated & Absent in normal \\
\hline SF3B1 & $\operatorname{chr} 2: 198265515 \mathrm{G}>\mathrm{C}$ & NM_012433 & c. $2642 \mathrm{C}>\mathrm{G}$ & p.A881G & P85 & Validated & No matched normal \\
\hline STK11 & chr19:1206917A $>C$ & NM_000455 & c. $5 \mathrm{~A}>\mathrm{C}$ & p.E2A & P85 & Validated & No matched normal \\
\hline TBX3 & chr12:115118738insT & NM_005996 & c.602_603insA & p.V202Rfs*38 & P54 & Validated & No matched normal \\
\hline TP53 & $\begin{array}{l}\text { chr17:7577024-7577048delTTAGTGC } \\
\text { TCCCTGGGGGCAGCTCGT }\end{array}$ & NM_000546.5 & $\begin{array}{l}\text { c.890_914delACGAGCTGCCC } \\
\text { CCAGGGAGCACTAA }\end{array}$ & p.H297Rfs* 40 & P56 & Validated & Absent in normal \\
\hline TP53 & chr17:7577105G $>A$ & NM 000546.5 & c. $833 \mathrm{C}>\mathrm{T}$ & p.P278L & P19 & Validated & No matched normal \\
\hline TP53 & chr17:7577131G $>C$ & NM_000546.5 & c. $807 \mathrm{C}>\mathrm{G}$ & p.S269R & P43 & Validated & Absent in normal \\
\hline TP53 & chr17:7577156C $>$ A & NM_000546.5 & c. $783-1 \mathrm{G}>\mathrm{T}$ & splice site & P27 & Validated & No matched normal \\
\hline TP53 & $\operatorname{chr} 17: 7577610 \mathrm{~T}>\mathrm{G}$ & NM_000546.5 & c. $673-2 \mathrm{~A}>\mathrm{C}$ & splice site & P44 & Validated & Absent in normal \\
\hline TP53 & $\operatorname{chr} 17: 7578496 A>G$ & NM_000546.5 & c. $434 \mathrm{~T}>\mathrm{C}$ & p.L145P & P54 & Validated & No matched normal \\
\hline TSC2 & $\operatorname{chr} 16: 2134485 G>A$ & NM_000548 & c. $4262 \mathrm{G}>\mathrm{A}$ & p.G1421E & P79 & Validated & No matched normal \\
\hline TSC2 & chr16:2134623C > T & NM_000548 & c. $4400 \mathrm{C}>\mathrm{T}$ & p.A1467V & P81 & Validated & No matched normal \\
\hline
\end{tabular}


a

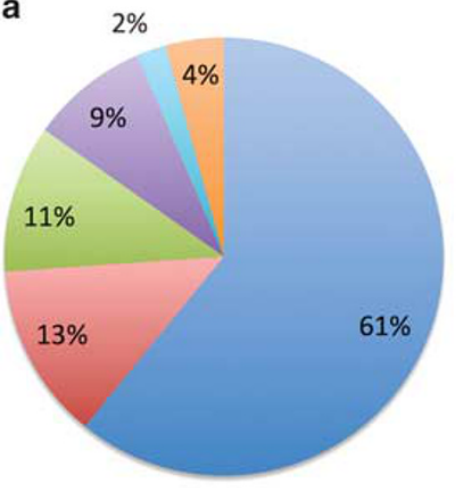
Missense
Splice site
- Frameshift insertion
a Frameshift deletion
= In-frame deletion
Nonsense

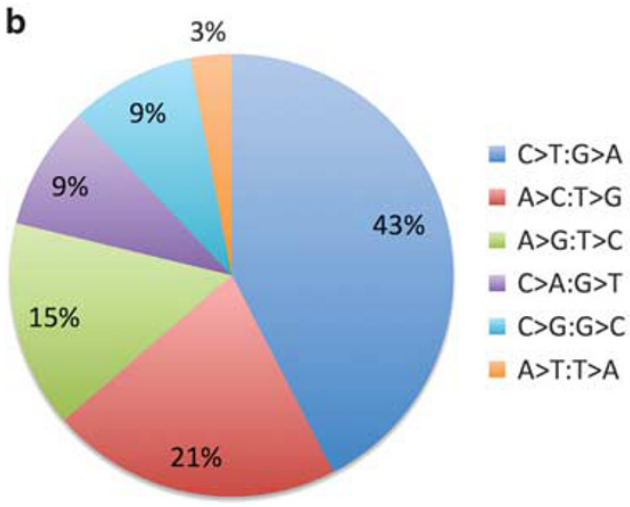

Figure 1 Mutation types in DCIS. (a) Distribution of mutation types. (b) Distribution of single nucleotide substitutions. Both consider only filtered variants; known germline variants excluded.

was limited, with only seven events and no cases of invasive recurrence.

\section{Mutations and DCIS Phenotype}

There was no difference in the median number of mutations or the median number of genes mutated by nuclear grade, ER status, PR status, or HER2 amplification status (Table 3). However, mutations of some genes did show an association with DCIS phenotype. TP53 mutations occurred exclusively in high nuclear grade DCIS $(P=0.011)$ and occurred more frequently in PR-negative DCIS (4/6, 66.7\%) compared with PR-positive DCIS (2/14, 14.3\%) $(P=0.037)$. There was a trend of TP53 mutations occurring more frequently in ER-negative DCIS cases but this association did not reach statistical significance $(3 / 4,75 \%$ ER-negative vs $3 / 16,18.8 \%$ ERpositive DCIS, $P=0.061)$. No association was observed between the presence of PIK3CA mutations and DCIS phenotype. Mutations of other genes occurred in too few cases for a meaningful assessment of association with phenotypic features.

\section{Copy Number Alterations}

We undertook genome-wide copy number analysis using the CopywriteR algorithm, which uses the off- target reads to estimate copy number. ${ }^{25}$ Two cases, P13 and P92, gave poor quality copy number output and were excluded from further analysis. All the remaining 18 cases had at least one copy number event, and the copy number profiles generated were similar to those we previously reported for pure DCIS $^{32}$, including common gains on $1 \mathrm{q}, 8 \mathrm{q}, 17 \mathrm{q}$, and 20q and frequent losses on 8p, 11q, 16q, and $17 \mathrm{p}$. There was good correlation between the copy number values generated by the on- and off-target reads for the genes in the panel (Spearman $r=0.96$, $P<0.0001$, Figure 4), and ERBB2 amplifications detected by sequencing were consistent with SISH data.

Copy number profiles were evaluated for various measures of genomic instability, including overall aberration type, the fraction of the genome altered by copy number and a measure of homologous recombination deficiency, the number of telomeric allelic imbalances. ${ }^{33}$ Seven cases had simple profiles (all were low or intermediate grade and ER positive), three cases had a background of simple copy number change but with two or more high-level amplifications, and the remaining eight cases had highly complex profiles, most with high level amplifications. No cases had chromothripsis. The fraction of the genome altered by copy number ranged from 2 to 47\% (median 12.9\%). TP53 mutant tumors had a significantly higher fraction of the genome altered by copy number than wild-type tumors (Figure 4, $P=0.005$, Mann-Whitney two-tail test). The median telomeric imbalance score for the 18 DCIS cases was 8 (range 0-22), slightly lower than the median of 12 in invasive breast cancer. ${ }^{34}$ In our previous cohort of 53 DCIS with molecular inversion probe array data, the median was 8.5 , thus we do not think the score is reduced by not having allelic imbalance information available. The telomeric imbalance score was significantly associated with TP53 mutation (Figure 4, $P=0.001)$ and high nuclear grade $(P=0.01)$.

We integrated our copy number and mutation data to investigate associations of particular mutations with copy number profiles. All TP53 mutant tumors had a complex copy number profile and were significantly enriched for gain of ERBB2, 3q, and $20 q$ and loss of 9p, 17p, and X (Figure 4). In invasive breast cancers from TCGA, 3q gain was also strongly associated with TP53 mutation but the other regions were not significant at a threshold of $>25 \%$ difference in frequency. All TP53 mutant tumors had either amplification $(n=4)$ or gain $(n=2)$ of ERBB2, similar to Abba et $a 1^{14}$ (amplified $n=4$, gain $n=1$ ). To further investigate this association, we compared p53 immunohistochemistry with ERBB2 amplification status by silver in situ hybridization in a wider DCIS cohort, using tissue microarrays including both pure DCIS $(n=187)$ and DCIS associated with invasive breast cancer $(n=19)$. Abnormal p53 staining was observed in 68/187 $(36.0 \%)$ pure DCIS and 9/19 (47\%) mixed DCIS, with the majority of abnormal cases (57\%) showing 


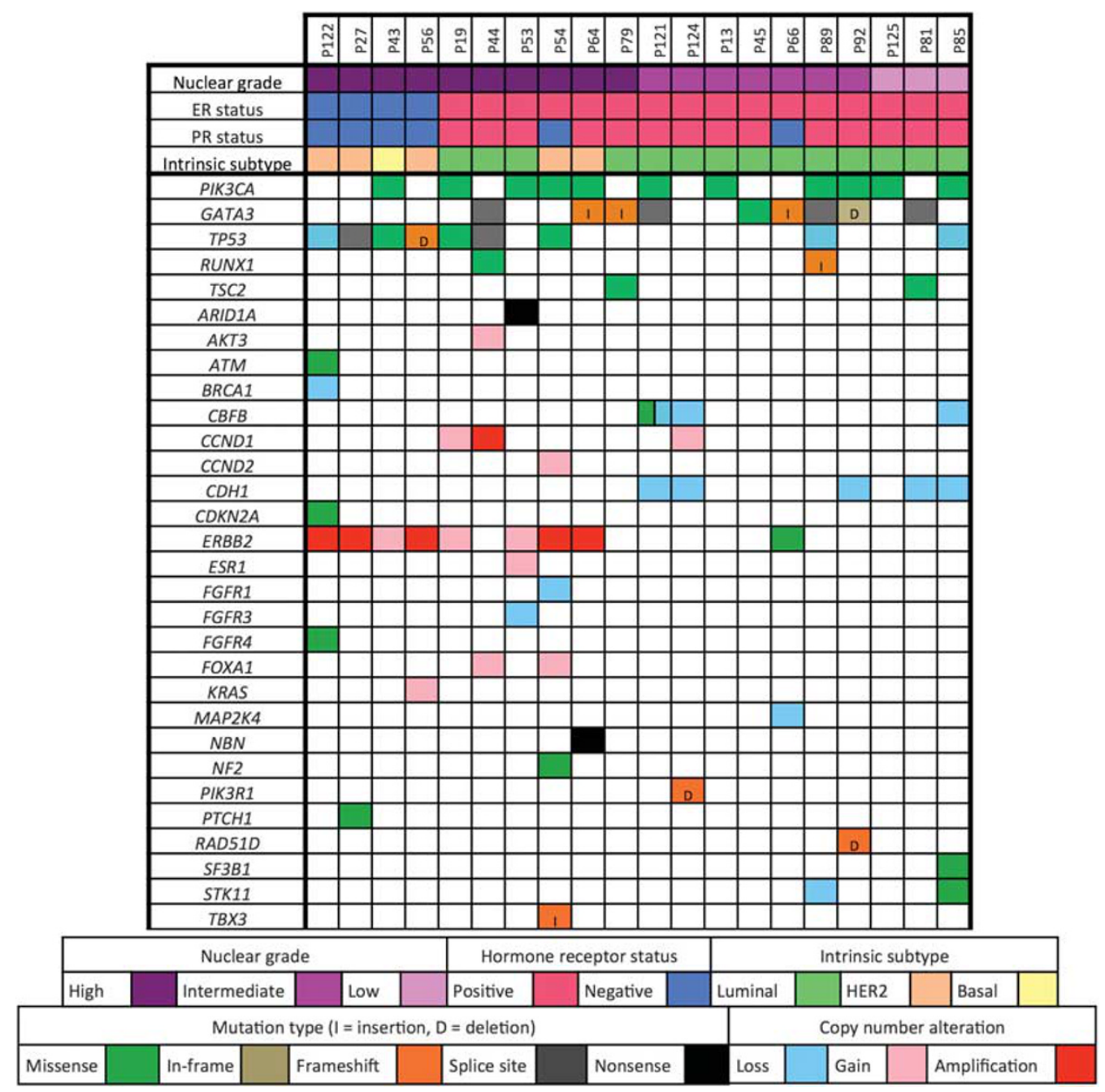

Figure 2 Mutations and copy number alterations identified in DCIS (germline variants excluded).

over-expression (Table 4). There was no significant difference in p53 positivity between pure and mixed DCIS $(P=0.46)$. A significant association of p53 abnormal staining with ERBB2 amplification was observed in pure DCIS $(P=0.033)$. Half of ERBB2 amplified pure DCIS cases were p53 abnormal, compared with $30 \%$ of ERBB2 non-amplified. Considering only p53 over-expression, $38 \%$ of ERBB2 amplified were p53 over-expressing, compared to $16 \%$ of ERBB2 non-amplified $(P=0.003)$. Abnormal p53 protein was also significantly associated with high nuclear grade, ER negativity, and PR negativity (Table 4).

Twelve of the twenty DCIS cases submitted for panel sequencing had p53 immunohistochemistry data. All five TP53 mutants with protein data were classified as p53 abnormal (either entirely negative for a truncating mutation or strongly positive in $>60 \%$ of cells for a missense mutation; sensitivity $100 \%$ ). Six of the seven cases classified as TP53 wildtype by sequencing were p53 normal by immunohistochemistry (specificity $85.7 \%$ ), with the one discordant case negative for protein. Immunohistochemistry is therefore a fair proxy for mutation status in DCIS using these thresholds, given likely tumor heterogeneity.

PIK3CA-mutated tumors were more likely to have loss of chromosome 18, while GATA3-mutated tumors were less likely to have loss of $8 \mathrm{p}$. Neither observation was significant in TCGA invasive breast cancer data. Neither PIK3CA nor GATA3 mutations were associated with a significant difference in the fraction of the genome altered by copy number or the number of telomeric imbalances.

\section{Discussion}

Little is known about the mutations that occur in DCIS. We therefore used massively parallel sequencing of a targeted panel of 107 cancer-related genes to determine mutations and copy number alterations in 20 DCIS cases of various phenotypes. The panel was selected to represent frequently mutated breast 
cancer drivers and clinically actionable genes. The technique was shown to be robust for the detection of genomic alterations in formalin-fixed paraffin
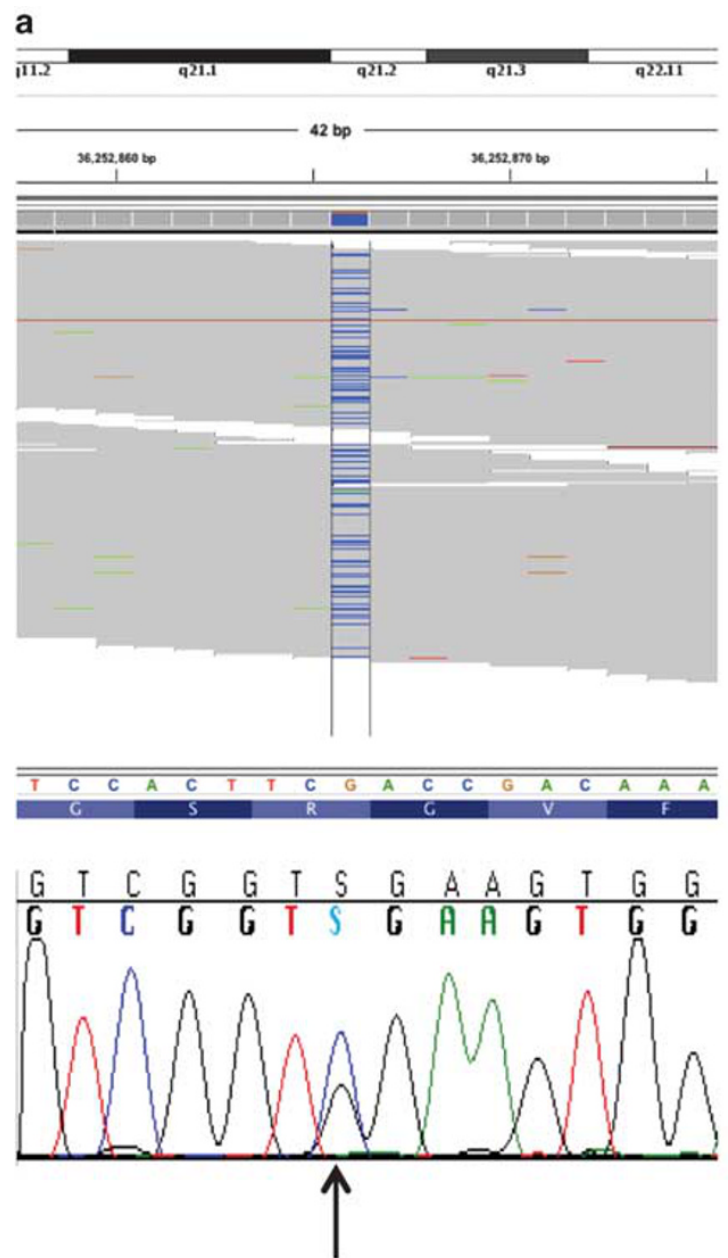

embedded derived DNA (96.0\% validation rate). Previous studies examining mutations of a broad panel of genes in DCIS have utilized DNA from

b
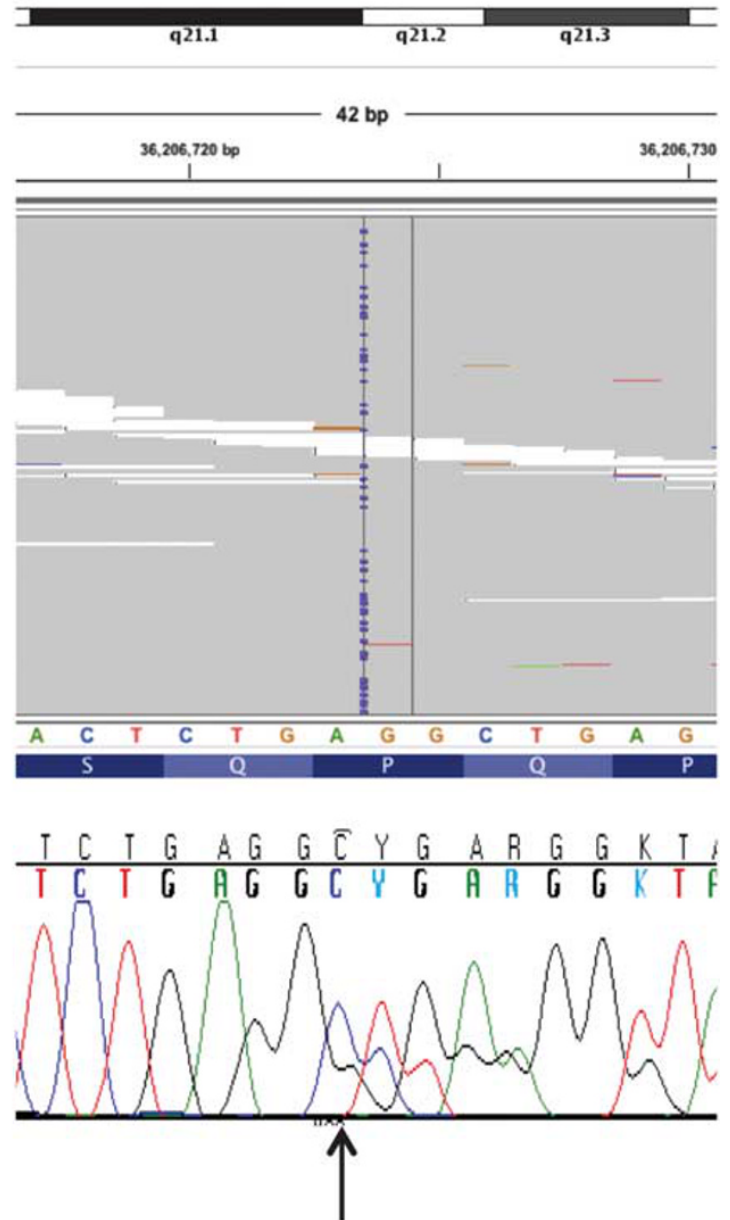

Figure 3 RUNX1 mutations. (a) Sample P44 with chr21:36252866G > C. (b) Sample P89 with chr21:36206724 insG. In both panels the Integrated Genome Viewer plot of the targeted sequencing bam file is shown at top, while the Sanger sequencing validation electrophoretogram is shown below.

Table 3 Associations between mutations and DCIS phenotype

\begin{tabular}{|c|c|c|c|c|c|c|c|c|c|c|c|c|}
\hline & $\begin{array}{l}\text { High } \\
\text { grade }\end{array}$ & $\begin{array}{l}\text { Non-high } \\
\text { grade }\end{array}$ & $\mathrm{P}$-value & ER+ & $E R-$ & P-value & $P R+$ & $P R-$ & $\mathrm{P}$-value & $\begin{array}{l}\text { HER2 } \\
\text { amplified }\end{array}$ & $\begin{array}{l}\text { HER2 non- } \\
\text { amplified }\end{array}$ & $\mathrm{P}$-value \\
\hline $\begin{array}{l}\text { Median number of } \\
\text { mutations per case }\end{array}$ & 2 & 2 & 0.971 & 2 & 2 & 0.554 & 2 & 2 & 0.968 & 2 & 2 & 0.800 \\
\hline $\begin{array}{l}\text { Median number of } \\
\text { mutated genes per case } \\
\text { GATA3 }\end{array}$ & 2 & 2 & 0.579 & 2 & 2 & 0.750 & 2 & 2 & 0.718 & 2 & 2 & 0.612 \\
\hline Mutation present & 3 & 6 & 0.370 & 9 & 0 & 0.094 & 8 & 1 & 0.157 & 1 & 8 & 0.319 \\
\hline No mutation & 7 & 4 & & 7 & 4 & & 6 & 5 & & 4 & 7 & \\
\hline \multicolumn{13}{|l|}{ PIКЗСА } \\
\hline Mutation present & 5 & 6 & 1.000 & 10 & 1 & 0.285 & 9 & 2 & 0.336 & 2 & 9 & 0.617 \\
\hline No mutation & 5 & 4 & & 6 & 3 & & 5 & 4 & & 3 & 6 & \\
\hline \multicolumn{13}{|l|}{ TP53 } \\
\hline Mutation present & 6 & 0 & 0.011 & 3 & 3 & 0.061 & 2 & 4 & 0.037 & 3 & 3 & 0.131 \\
\hline No mutation & 4 & 10 & & 13 & 1 & & 12 & 2 & & 2 & 12 & \\
\hline
\end{tabular}

Bold values indicate statistical significance at $P<0.05$. 

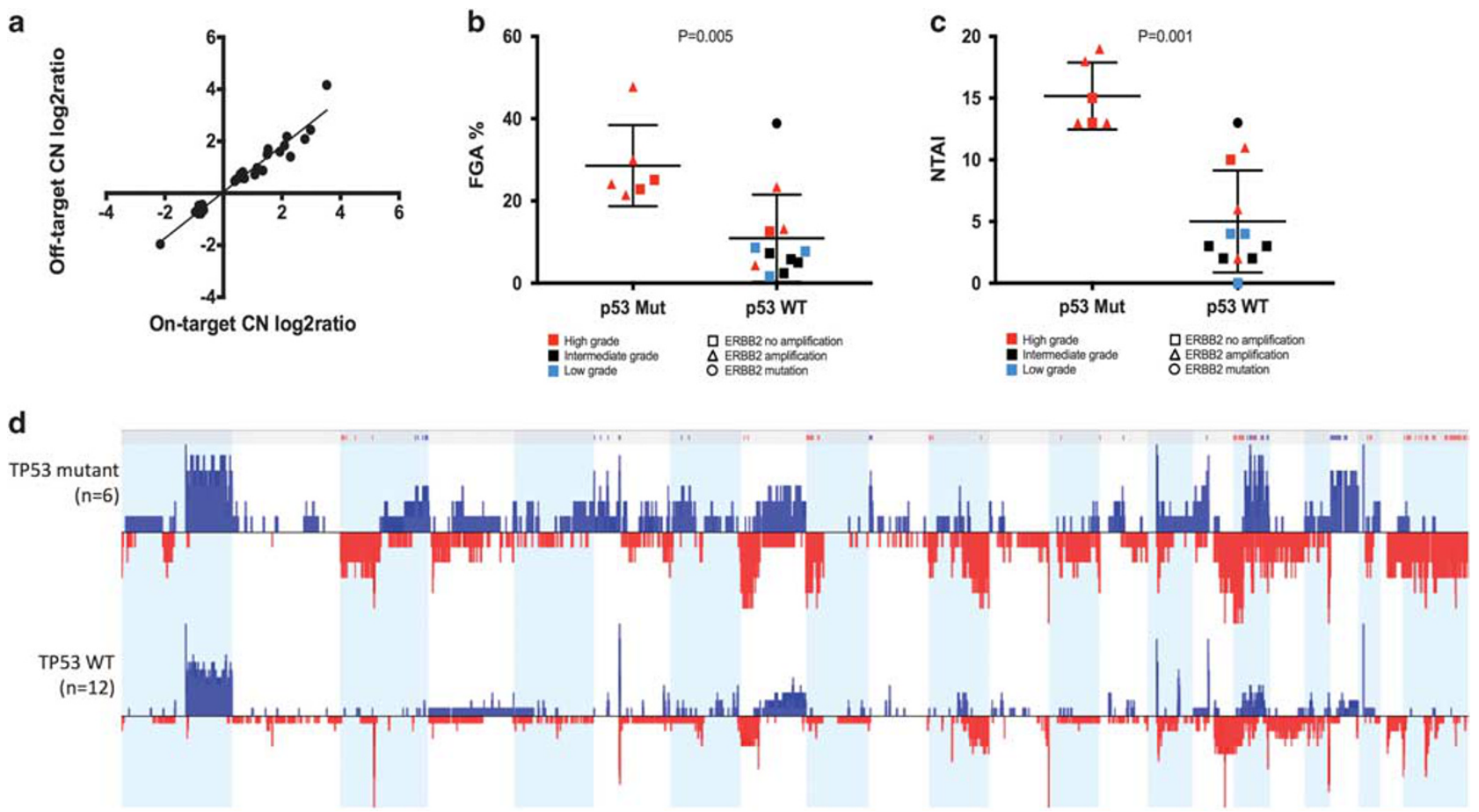

Figure 4 Integration of mutation with copy number data. (a) Good correlation between on- and off-target copy number predictions was observed. (b) Fraction of the genome altered by copy number (FGA) by TP53 mutation status. (c) Number of telomeric imbalances (NTAI) by TP53 mutation status. (d) Comparison of copy number profiles of TP53 mutant vs wild-type tumors (blue= gain, red=loss). Bar at the top indicates statistical significance at $P<0.05$.

fresh-frozen tumor specimens, but this study shows that interrogation of formalin-fixed paraffin embedded-derived DNA can now be applied to a variety of DCIS cases from hospital-based series, including cases archived for up to nine years at the time of DNA extraction.

The rate of variants in DCIS samples was higher (5.6 mutations/Mb) than that previously reported for DCIS (1.61 mutations/Mb) ${ }^{14}$ and invasive breast cancer (1.66 mutations/Mb) ${ }^{4}$ and is likely to be due to the use of a panel focussed on genes known to be mutated in breast cancer. Although potential germline variants might have contributed to this (these were unable to be completely excluded as few cases had matched normal DNA available), the number of these germline variants is expected to be small, as stringent population filters based on large public databases were applied.

The most prevalent single-base substitution observed was the $\mathrm{C}>\mathrm{T}: \mathrm{G}>\mathrm{A}$ alteration, accounting for $43 \%$ of single-nucleotide substitutions detected. The $\mathrm{C}>\mathrm{T}: \mathrm{G}>\mathrm{A}$ alteration forms a major part of three of the five mutational signatures identified in breast cancer ${ }^{35}$ and is thought to occur through the spontaneous deamination of 5-methyl-cytosine which accumulates with age ${ }^{35,36}$ and overactivity of the APOBEC family of cytidine deaminases. ${ }^{35,37}$ Although $\mathrm{C}>\mathrm{T}: \mathrm{G}>\mathrm{A}$ alterations are also characteristic of formalin-related DNA damage ${ }^{18}$ the proportion of $\mathrm{C}>\mathrm{T}: \mathrm{G}>\mathrm{A}$ alterations is similar to that reported in fresh-frozen samples of invasive breast cancer $(\sim 40 \%)^{4}$ and DCIS $(\sim 50 \%)^{14,15}$, so the $\mathrm{C}>\mathrm{T}$ : $\mathrm{G}>\mathrm{A}$ alterations detected are unlikely to represent formalin-fixation artefacts.

Mutations of 19 genes were identified including those involved in DNA repair and cell cycle control (ARID1A, ATM, CDKN2A, NBN, RAD51D, TP53), the PI3K/AKT/mTOR pathway (PIK3CA, PIK3R1, TSC2), the Hedgehog pathway (PTCH1), transcription factors (CBFB, GATA3, RUNX1, TBX3), receptor tyrosine kinases (ERBB2, FGFR4), serine/threonine-protein kinases (STK11), other tumor suppressor genes (NF2), and splicing factors (SF3B1), suggesting that multiple mechanisms are disrupted in the development of DCIS. Notably, unlike Abba et al, ${ }^{14}$ we only saw one case that had no common driver (ie, wildtype for TP53, PIK3CA, ERBB2, and GATA3). Although this case (P124) had a relatively stable genome ( $5 \%$ affected by copy number), the copy number changes included a CCND1 amplification, $C B F B$ loss, and $C D H 1$ loss. The sample also carried two PIK3R1 truncating mutations. Thus, DCIS cases of all phenotypes carry mutational drivers reminiscent of invasive breast cancer, although the frequency of some events, such as GATA3 mutations, may vary. Analysis of more DCIS cases with matching normal DNA will be required to determine whether the more rarely mutated genes are indeed more frequently affected in DCIS than invasive breast cancer.

The prevalence of PIK3CA mutations in this cohort $(55 \%)$ is somewhat higher than that previously 
Table 4 p53 immunohistochemistry

\begin{tabular}{|c|c|c|c|c|c|c|}
\hline & $\begin{array}{l}\text { Pure DCIS } \\
(\mathrm{n}=187)\end{array}$ & \multirow[b]{2}{*}{ p53 normal } & \multirow[b]{2}{*}{$\begin{array}{c}\text { P-value } \\
\text { (Fisher's exact test) }\end{array}$} & \multirow{2}{*}{$\begin{array}{c}\begin{array}{c}\text { Mixed DCIS } \\
(\mathrm{n}=19)\end{array} \\
\text { p53 abnormal }\end{array}$} & \multirow[b]{2}{*}{ p53 normal } & \multirow[b]{2}{*}{$\begin{array}{c}\mathrm{P}-\text { value } \\
\text { (Fisher's exact test) }\end{array}$} \\
\hline & p53 abnormal & & & & & \\
\hline \multicolumn{7}{|l|}{ Nuclear grade } \\
\hline High grade & 43 & 55 & $<0.001$ & 8 & 3 & 0.004 \\
\hline Non-high grade & 13 & 56 & & 0 & 7 & \\
\hline \multicolumn{7}{|l|}{ ER status } \\
\hline ER positive & 30 & 90 & $<0.0001$ & 3 & 8 & 0.11 \\
\hline ER negative & 32 & 24 & & 4 & 1 & \\
\hline \multicolumn{7}{|l|}{$P R$ status } \\
\hline PR positive & 17 & 74 & $<0.0001$ & 0 & 5 & 0.044 \\
\hline PR negative & 43 & 43 & & 6 & 4 & \\
\hline \multicolumn{7}{|l|}{ HER2 status } \\
\hline Amplified & 19 & 19 & 0.033 & 3 & 2 & 0.62 \\
\hline Non-amplified & 42 & 99 & & 5 & 7 & \\
\hline \multicolumn{7}{|l|}{ Clinical outcome } \\
\hline Recurred & 3 & 9 & 0.65 & - & - & \\
\hline No recurrence & 4 & 6 & & & & \\
\hline
\end{tabular}

reported for DCIS (17-48\% $)^{7,8,14,15,38,39}$ and invasive breast cancers (25-36\%). ${ }^{4,5,40,41}$ The relatively small number of cases included in this and other DCIS each studies (involving between six and 202 cases), ${ }^{7,8,14,15,38,39}$ may account for the variation in prevalence. Alternatively, the high read-depth of our targeted sequencing may have led to increased detection rates compared with exome or Sanger studies: four DCIS cases had a PIK3CA variant frequency of $<20 \%$, which could have been missed by less sensitive methods. The role of PIK3CA mutations as markers of tumor progression in DCIS is uncertain. PIK3CA mutations in invasive breast cancers have been associated with favorable tumor features $^{41}$ and better prognosis in patients with ERpositive, HER2-negative tumors; 40 however, we saw no association of PIK3CA mutations with clinicopathological features in our cohort.

The GATA3 mutations were mostly observed in ER-positive cases (93\%), in keeping with the reported association of GATA3 mutations with luminal breast cancer. ${ }^{5,42}$ Only one case had both GATA3 and TP53 mutations, reflecting a pattern reported in invasive breast tumors where mutations of these two genes rarely co-exist. ${ }^{42}$ Four DCIS cases had both GATA3 and PIK3CA mutations, in contrast to invasive carcinomas in which mutations of these two genes are almost mutually exclusive. ${ }^{1,42}$ In one case the allele frequencies suggest that the GATA3 and PIK3CA mutations could be present in different clones.

The prevalence of GATA3 mutations in the current DCIS cohort is significantly higher than that reported in invasive breast cancer $(4-22 \%)^{4,5,30,31}$ and in DCIS. Recently, Abba et al ${ }^{14}$ reported a GATA3 mutation rate of $7 \%$ in 30 pure high grade DCIS cases while Kim et al ${ }^{15}$ observed GATA3 mutation in one low grade DCIS out of a cohort of in six DCIS lesions of various nuclear grades $(16.7 \%)$. If the prevalence of GATA3 mutations observed in the current cohort is truly reflective of the general DCIS population, the high prevalence of GATA3 mutations in DCIS compared with invasive carcinomas suggests that GATA3 mutations, like HER2 amplification, are not selected for during the transition to invasive disease and perhaps may indicate better prognosis, similar to that observed in invasive carcinomas. ${ }^{42}$ Our extended cohort did not have sufficient invasive recurrences to be able to test this hypothesis.

TP53 mutations were present in exclusively in high grade DCIS, and half the mutations were missense; both these features are consistent with previous reports of TP53 mutations in both invasive and in situ breast cancer. ${ }^{1,4-6,12,13}$ We also noted an association of abnormal p53 protein with ERBB2 amplification. In invasive breast cancer from TCGA, TP53 mutation is also significantly associated with ERBB2 amplification $(P<0.0001)$, and the proportion of ERBB2 amplified cases with TP53 mutation $(61 / 118,52 \%)$ is similar to our pure DCIS cohort $(50 \%)$, despite the different methods used (exome vs immunohistochemistry and SNP arrays vs in situ hybridization for p53 and $E R B B 2$, respectively). A key question in DCIS biology is the relatively higher proportion of cases with ERBB2 amplification compared to invasive cases: our data would not support a role for TP53 in explaining this difference. 
We identified RUNX1 mutations in two DCIS cases, which has not previously been reported in DCIS but occurs in $4-5 \%$ of invasive breast carcinomas, ${ }^{4,5}$ where mutations are associated with luminal B expression profiles and high grade tumors ${ }^{1}$. The DCIS cases with RUNX1 mutations were both ER positive, HER2 negative and high or intermediate grade, consistent with a luminal B phenotype. RUNX1 is required for ER localization and regulation of target genes ${ }^{43}$ and is believed to function as a tumor suppressor gene in breast cancer. ${ }^{44}$ We also observed a mutation of $C B F B$ which was mutually exclusive with RUNX1 mutation, consistent with their role as subunits of a heterodimeric transcription factor. The CBFBmutated case was also ER positive, HER2 negative and intermediate grade. The mutually exclusive relationship between mutations of $R U N X 1$ and $C B F B$, and also PIK3R1 and PIK3CA is observed in invasive breast cancer. ${ }^{4,5}$

As the panel assays genes predominantly altered in invasive breast cancer rather than an agnostic exome or genome approach, mutations unique to DCIS were unable to be identified. Nevertheless, a targeted approach has several advantages, including high read-depth, which enabled detection of mutations even at allele frequencies of $10 \%$. In addition, the cost was relatively low and bioinformatics analysis quick and straightforward. We were also able to obtain good quality copy number data from almost all samples, which enabled copy number drivers such as CCND1 amplification and MAP2K4 homozygous deletion to be observed. Copy number alterations of MAP2K4 have not previously been reported in DCIS.

In conclusion, this study provides a snapshot of the mutational profiles of DCIS, incorporating both copy number and somatic point mutations. The entire spectrum of mutations in DCIS and even in an individual tumor is unlikely to have been documented in this study due to the small cohort, targeted gene panel approach and limited sampling of the lesions. Nonetheless, mutations were present in all samples suggesting that mutational processes have a role in DCIS biology. While generally similar mutational patterns to invasive breast carcinomas were observed, there was a surprisingly high prevalence of GATA3 mutations. Given that GATA3 mutations are associated with improved survival in patients with invasive breast cancer, it is hypothesized that GATA3 mutations in DCIS may be a marker of less aggressive behavior. In contrast, TP53 mutations were associated with adverse tumor characteristics. The role of copy number alteration, telomeric imbalance and specific gene mutations as prognostic biomarkers will require testing in a suitable cohort of DCIS with outcome data available. The panel testing approach used here will be an appropriate methodology to address these questions.

\section{Acknowledgments}

This study was supported by the Australian National Health and Medical Research Council (ID \#50950, \#566603, and APP1063092) and the National Breast Cancer Foundation (NBCF). TK was supported by a Melbourne University International Scholarship. We thank Dr Siobhan Hughes for technical assistance.

\section{Disclosure/conflict of interest}

The authors declare no conflict of interest.

\section{References}

1 Ellis MJ, Ding L, Shen D, et al. Whole-genome analysis informs breast cancer response to aromatase inhibition. Nature 2012;486:353-360.

2 Curtis C, Shah SP, Chin SF, et al. The genomic and transcriptomic architecture of 2,000 breast tumours reveals novel subgroups. Nature 2012;486:346-352.

3 Stephens PJ, McBride DJ, Lin ML, et al. Complex landscapes of somatic rearrangement in human breast cancer genomes. Nature 2009;462:1005-1010.

4 Banerji S, Cibulskis K, Rangel-Escareno C, et al. Sequence analysis of mutations and translocations across breast cancer subtypes. Nature 2012;486:405-409.

5 Cancer Genome Atlas N. Comprehensive molecular portraits of human breast tumours. Nature 2012;490: 61-70.

6 Mao X, Fan C, Wei J, et al. Genetic mutations and expression of p53 in non-invasive breast lesions. Mol Med Rep 2010;3:929-934.

7 Sakr RA, Weigelt B, Chandarlapaty S, et al. PI3K pathway activation in high-grade ductal carcinoma in situ-implications for progression to invasive breast carcinoma. Clin Cancer Res 2014;20:2326-2337.

8 Miron A, Varadi M, Carrasco D, et al. PIK3CA mutations in in situ and invasive breast carcinomas. Cancer Res 2010;70:5674-5678.

9 Dunlap J, Le C, Shukla A, et al. Phosphatidylinositol3-kinase and AKT1 mutations occur early in breast carcinoma. Breast Cancer Res Treat 2010;120:409-418.

10 Munn KE, Walker RA, Menasce L, et al. Mutation of the TP53 gene and allelic imbalance at chromosome 17 p13 in ductal carcinoma in situ. Br J Cancer 1996;74: 1578-1585.

11 Chitemerere M, Andersen TI, Holm R, et al. TP53 alterations in atypical ductal hyperplasia and ductal carcinoma in situ of the breast. Breast Cancer Res Treat 1996;41:103-109.

12 Done SJ, Eskandarian S, Bull S, et al. p53 missense mutations in microdissected high-grade ductal carcinoma in situ of the breast. J Natl Cancer Inst 2001;93: 700-704.

13 Zhou W, Muggerud AA, Vu P, et al. Full sequencing of TP53 identifies identical mutations within in situ and invasive components in breast cancer suggesting clonal evolution. Mol Oncol 2009;3:214-219.

14 Abba MC, Gong T, Lu Y, et al. A molecular portrait of high-grade ductal carcinoma in situ. Cancer Res 2015;75:3980-3990.

15 Kim SY, Jung SH, Kim MS, et al. Genomic differences between pure ductal carcinoma in situ and 
synchronous ductal carcinoma in situ with invasive breast cancer. Oncotarget 2015;6:7597-7607.

16 Gorringe K, Hunter S, Pang J, et al. Copy number analysis of DCIS with and without recurrence. Mod Pathol 2015;28:1174-1184.

17 Li H, Durbin R. Fast and accurate short read alignment with Burrows-Wheeler transform. Bioinformatics 2009; 25:1754-1760.

18 Wong SQ, Li J, Tan AY, et al. Sequence artefacts in a prospective series of formalin-fixed tumours tested for mutations in hotspot regions by massively parallel sequencing. BMC Med Genomics 2014;7:23.

19 Genomes Project C, Abecasis GR, Auton A, et al. An integrated map of genetic variation from 1,092 human genomes. Nature 2012;491:56-65.

20 Untergasser A, Cutcutache I, Koressaar T, et al. Primer3-new capabilities and interfaces. Nucleic Acids Res 2012;40:e115.

21 Koressaar T, Remm M. Enhancements and modifications of primer design program Primer3. Bioinformatics 2007;23:1289-1291.

22 Pang JM, Deb S, Takano EA, et al. Methylation profiling of ductal carcinoma in situ and its relationship to histopathological features. Breast Cancer Res 2014; 16:423.

23 Zardawi SJ, Zardawi I, McNeil CM, et al. High Notch1 protein expression is an early event in breast cancer development and is associated with the HER-2 molecular subtype. Histopathology 2010;56:286-296.

24 Yemelyanova A, Vang R, Kshirsagar M, et al. Immunohistochemical staining patterns of p53 can serve as a surrogate marker for TP53 mutations in ovarian carcinoma: an immunohistochemical and nucleotide sequencing analysis. Mod Pathol 2011;24:1248-1253.

25 Kuilman T, Velds A, Kemper K, et al. CopywriteR: DNA copy number detection from off-target sequence data. Genome Biol 2015;16:49.

26 Cerami E, Gao J, Dogrusoz U, et al. The cBio cancer genomics portal: an open platform for exploring multidimensional cancer genomics data. Cancer Discov 2012;2:401-404.

27 Kumar P, Henikoff S, Ng PC. Predicting the effects of coding non-synonymous variants on protein function using the SIFT algorithm. Nat Protoc 2009;4: 1073-1081.

28 Choi Y, Sims GE, Murphy S, et al. Predicting the functional effect of amino acid substitutions and indels. PLoS ONE 2012;7:e46688.

29 Petitjean A, Mathe E, Kato S, et al. Impact of mutant p53 functional properties on TP53 mutation patterns and tumor phenotype: lessons from recent developments in the IARC TP53 database. Hum Mutat 2007;28: 622-629.

30 Arnold JM, Choong DY, Thompson ER, et al. Frequent somatic mutations of GATA3 in non-BRCA1/BRCA2 familial breast tumors, but not in BRCA1-, BRCA2or sporadic breast tumors. Breast Cancer Res Treat 2010;119:491-496.

31 Usary J, Llaca V, Karaca G, et al. Mutation of GATA3 in human breast tumors. Oncogene 2004;23:7669-7678.
32 Gorringe KL, Hunter SM, Pang JM, et al. Copy number analysis of ductal carcinoma in situ with and without recurrence. Mod Pathol 2015;28:1174-1184.

33 Birkbak NJ, Wang ZC, Kim JY, et al. Telomeric allelic imbalance indicates defective DNA repair and sensitivity to DNA-damaging agents. Cancer Discov 2012;2: 366-375.

34 Marquard AM, Eklund AC, Joshi T, et al. Pan-cancer analysis of genomic scar signatures associated with homologous recombination deficiency suggests novel indications for existing cancer drugs. Biomark Res 2015;3:9.

35 Alexandrov LB, Nik-Zainal S, Wedge DC, et al. Signatures of mutational processes in human cancer. Nature 2013;500:415-421.

36 Pfeifer GP. Mutagenesis at methylated CpG sequences. Curr Top Microbiol Immunol 2006;301:259-281.

37 Nik-Zainal S, Alexandrov LB, Wedge DC, et al. Mutational processes molding the genomes of 21 breast cancers. Cell 2012;149:979-993.

38 Ang DC, Warrick AL, Shilling A, et al. Frequent phosphatidylinositol-3-kinase mutations in proliferative breast lesions. Mod Pathol 2014;27:740-750.

39 Li H, Zhu R, Wang L, et al. PIK3CA mutations mostly begin to develop in ductal carcinoma of the breast. Exp Mol Pathol 2010;88:150-155.

40 Loi S, Haibe-Kains B, Majjaj S, et al. PIK3CA mutations associated with gene signature of low mTORC1 signaling and better outcomes in estrogen receptor-positive breast cancer. Proc Natl Acad Sci USA 2010;107: 10208-10213.

41 Kalinsky K, Jacks LM, Heguy A, et al. PIK3CA mutation associates with improved outcome in breast cancer. Clin Cancer Res 2009;15:5049-5059.

42 Jiang YZ, Yu KD, Zuo WJ, et al. GATA3 mutations define a unique subtype of luminal-like breast cancer with improved survival. Cancer 2014;120:1329-1337.

43 Stender JD, Kim K, Charn TH, et al. Genome-wide analysis of estrogen receptor alpha DNA binding and tethering mechanisms identifies Runx1 as a novel tethering factor in receptor-mediated transcriptional activation. Mol Cell Biol 2010;30:3943-3955.

44 Janes KA. RUNX1 and its understudied role in breast cancer. Cell Cycle 2011;10:3461-3465.

This work is licensed under a Creative Commons Attribution-NonCommercialNoDerivs 4.0 International License. The images or other third party material in this article are included in the article's Creative Commons license, unless indicated otherwise in the credit line; if the material is not included under the Creative Commons license, users will need to obtain permission from the license holder to reproduce the material. To view a copy of this license, visit http://creativecommons.org/licenses/ by-nc-nd/4.0/

(c) The Author(s) 2017

Supplementary Information accompanies the paper on Modern Pathology website (http://www.nature.com/ modpathol) 